\title{
Chlorambucil/Obinutuzumab Regimen
}

National Cancer Institute

\section{Source}

National Cancer Institute. ChlorambucillObinutuzumab Regimen. NCI Thesaurus. Code C160522.

A regimen consisting of chlorambucil and obinutuzumab that can be used for the treatment of chronic lymphocytic leukemia (CLL). 